\title{
ALTERNATIVAS ORGÁNICAS PARA LA DESINFECCIÓN DE SUELOS EN EL CULTIVO DE Gypsophila paniculata
}

\section{ORGANIC ALTERNATIVES FOR SOIL DISINFECTION IN THE CULTIVATION OF Gypsophila paniculata}

\author{
Jhonn Cueva ${ }^{(1)}$, Victor Abril(2), Mijail Nozhevnik(1) \\ (1) Instituto Agrotecnológico, Universidad de Rusia Amistad de los Pueblos, Rusia, \\ Moscú. e-mail: ihonnlenon@hotmai.es nozhevnik-mi@rudn.ru \\ (2) Departamento Ciencias de la Vida, Universidad de las Fuerzas Armadas - \\ ESPE, Sangolquí, Ecuador. e-mail: \\ vhabril@espe.edu.ec
}

Recibido: Abril, 2019 Aceptado: Noviembre, 2019

\section{RESUMEN}

Las alternativas orgánicas para desinfección de suelos, en los cultivos de flores de verano, posee gran importancia para el sector floricultor ecuatoriano, no solo por el costo de los métodos químicos convencionales sino por su impacto en la salud humana y el medio ambiente. El presente estudio consistió en comparar los siguientes tratamientos de desinfección en el cultivo de Gypsophila paniculata en: dos paquetes comerciales orgánicos, a base de microorganismos biocontroladores y biopreparados, de las empresas Bioseb (T1) y Agroinnovación (T2); tres tratamientos basados en la técnica de Biosolarización, con diferentes dosis de material orgánico; $2 \mathrm{~kg} \cdot \mathrm{m}^{-2}$ de material vegetal $+0.5 \mathrm{~kg} \cdot \mathrm{m}^{-2}$ de gallinaza (T3), $4 \mathrm{~kg} \cdot \mathrm{m}^{-}$ 2 de material vegetal $+2 \mathrm{~kg} \cdot \mathrm{m}^{-2}$ de gallinaza (T4), $5 \mathrm{~kg} \cdot \mathrm{m}^{-2}$ de gallinaza (T5) y un testigo químico Dazomet 98\% (T0). Mediante la evaluación de las variables productivas, se determina como mejor tratamiento: de Biosolarización (T3) y de los paquetes orgánicos comerciales (T1).

Palabras claves: Biopreparados, Biosolarización, Dazomet, Desinfección de Suelos, Mortalidad.

\section{ABSTRACT}

The organic alternatives for soil disinfection in summer flower crops, has great importance for the Ecuadorian floriculture sector, not only for the cost of conventional chemical methods, but for its impact on human health and the environment. The present study consisted of comparing the following disinfection treatments in the cultivation of Gypsophila paniculata: two organic commercial packages, based on biocontrol and bioprepared microorganisms of the companies 
Bioseb (T1) and Agroinnovación (T2); three treatments based on the technique of Biosolarization with different doses of organic material, $2 \mathrm{~kg} \cdot \mathrm{m}-2$ of plant material $+0.5 \mathrm{~kg} \cdot \mathrm{m}-2$ of chicken manure (T3), $4 \mathrm{~kg} \cdot \mathrm{m}-2$ of plant material $+2 \mathrm{~kg} \cdot \mathrm{m}-2$ of chicken manure (T4), $5 \mathrm{~kg} \cdot \mathrm{m}-2$ of chicken manure (T5) and a chemical control Dazomet $98 \%$ (T0). By means of the evaluation of the productive variables, it is determined as the best treatment: of Biosolarization (T3) and of the commercial organic packages (T1).

Keywords:. Biopreparations, Biosolarization, Dazomet, Soil Disinfection, Mortality.

\section{INTRODUCCIÓN}

Gypsophila es una flor de relleno utilizada en arreglos y decoraciones florales. Ecuador es el mayor productor de Gypsophilas en el mundo con una participación del $77 \%$ en la producción mundial y con un $14,2 \%$ del total de la superficie sembrada en florícolas del país. (Prochile, 2017).

La desinfección de suelos es una intervención en los sistemas de cultivo con el principal objetivo de mejora de calidad de los productos obtenidos, por tal razón en el cultivo de Gypsophila, se lo realiza con el principal fin de controlar los fitopatógenos de origen edáfico, que representan un limitante en su producción.

La forma habitual de superar esta limitación ha sido el empleo sistemático de productos químicos de síntesis, con mayor o menor eficacia en el control de los organismos patógenos edáficos, destacando por su importancia el bromuro de metilo (BM), el 1,3-dicloropropeno (1,3-D), 1,3-dicloropropeno + cloropicrina (1,3$\mathrm{D}+\mathrm{Pic}$ ), cloropicrina (Pic), dazomet y metam sodio (metam-Na), así como otros biocidas químicos, principalmente nematicidas, fungicidas del suelo o herbicidas (Barres et al. 2006). El fundamento de la desinfección de suelos o sustratos empleando productos químicos está basado en la capacidad que tienen dichos productos de pasar a estado gaseoso en el momento de ser liberados, haciéndose necesario impedir el escape de dichos gases al medio ambiente, reteniéndolos durante el tiempo necesario para que su acción resulte efectiva. Entre los métodos de control químico, como uno de los fumigantes de menor toxicidad, tenemos Dazomet 98\%, (nombre químico tetrahidro-3,5-dimetil-2H-1, 3,5-tiadiazin-2-tiona), el cual al ponerse en contacto con la superficie húmeda, la sustancia activa se descompone en metilisotiocianato, formaldehído, metilamina y sulfuro de hidrógeno. Posee eficacia contra los fitopatógenos transmitidos por el suelo como los hongos de los géneros Pythium, Phytophthora, Rhizoctonia, Verticillium, Fusarium, Phoma, Didymella, e incluyendo a los nematodos Pratylenchus sp., Rotylenchus sp., Meloidogyne sp., además de especies del género Heterodera, plagas - gusano cable, Melolontha melolontha y otros (Neshev, G., 2008). Al ser utilizado en el manejo convencional en la plantación donde se ejecutó el ensayo se lo uso como tratamiento testigo.

La alta toxicidad que poseen los métodos químicos para la desinfección de suelos, compromete la búsqueda y evaluación de alternativas orgánicas sostenibles, sustentables y ambientalmente amigables, no solo por la importancia económica del cultivo sino por su relación con la salud humana y el medio ambiente. La agricultura orgánica, es un sistema agrícola autónomo basado en la utilización 
óptima de los recursos naturales, sin emplear productos químicos de síntesis, u organismos genéticamente modificados (OGMs), a la vez que se conserva la fertilidad de la tierra y se respeta el medio ambiente, todo ello de manera sostenible y equilibrada (Oelhaf, 1978). Dentro de las alternativas orgánicas para el control de patógenos edáficos, existe el control biológico. Consiste en el empleo de organismos antagonistas, quienes gracias a los biocidas que producen, por competencia por el alimento o al parasitar directamente al patógeno, evitan que este último alcance poblaciones altas para causar enfermedades severas (Agrios 1998, Agrawal et al. 1999). Otra alternativa orgánica son los biopreparados. Sustancias y mezclas de origen vegetal, animal o mineral presentes en la naturaleza que tienen propiedades nutritivas para las plantas o repelentes y atrayentes de insectos para la prevención y control de plagas y/o enfermedades (Fao, 2010). Basados en estos principios de control bilógico y biopreparados se fundamentan los dos paquetes orgánicos comerciales de las empresas Bioseb y Agroinnovación a evaluarse. Una alternativa orgánica más es la Biosolarización. Definida como la acción de sustancias volátiles producidas en la biodegradación de la materia orgánica en el control de los patógenos de las plantas, aumentando la eficacia de la técnica cuando forma parte de un sistema de producción integrada, describe la metodología que una vez esparcido uniformemente, el biofumigante (material orgánico de origen animal o vegetal) debe ser incorporado de forma inmediata en el suelo y, tras un pase de rotovator, se riega el terreno, preferiblemente por aspersión, aunque se puede regar a manta o instalar goteros, hasta que el suelo queda saturado, una vez finalizado el proceso se cubre el terreno con plástico durante al menos dos semanas, evitando la salida de los gases producidos en la biodegradación de la materia orgánica (Tello y Bello 2002). Se evaluaron tres tratamientos basados en la técnica de Biosolarización con distintas dosis de material orgánico: vegetal (residuos de producción de Gypsophila) y animal (gallinaza).

\section{METODOLOGÍA}

Este estudio se realizó en la parroquia de Otón, provincia Pichincha, Ecuador, Finca Florícola FLODECOL S.A, especializada en Gypsophilas. Dentro de la investigación se compararon seis tratamientos con cuatro repeticiones, con un total de 24 unidades experimentales, cada una de forma rectangular (cama de cultivo + camino de $30 \mathrm{~m} \times 1,8 \mathrm{~m}$ ) con $54 \mathrm{~m}^{2}$, con una densidad de 8 plantas $\cdot \mathrm{m}^{-2}$, con 4 hileras de plantas y un área total del ensayo de $1296 \mathrm{~m}^{2}$, las unidades experimentales se organizaron en grupos según el tratamiento (6 grupos), con el fin de evitar sesgo por interacción entere tratamientos, a los que se les asigno una ubicación al azar dentro del área de experimento. Se seleccionó 50 plantas al azar por cada unidad experimental, para determinar las variables productivas evaluadas: número de brotes a producción por planta a los 40 días posterior al pinch, número de tallos por planta a la cosecha, mortalidad expresada en porcentaje, peso de tallos por planta a la cosecha. Para el análisis estadístico se realizó la prueba de Comparación de Medias, Método de la Diferencia Mínima Significativa (LSD) al 5\%, para la comparación entre tratamientos, mediante la utilización del programa estadístico R-project (Tinn-R). Los tratamientos evaluados fueron:

T0 testigo químico Dazomet (tetrahidro-3,5-dimetil-2H-1, 3,5-tiadiazin-2tiona). Se aplica un riego con aspersión durante 2 días, hasta alcanzar $60 \mathrm{~cm}$ de profundidad de humedad, requisito para aplicar Dazomet 98\%, con una dosis de 
$50 \mathrm{~g} \cdot \mathrm{m}^{-2}$ de manera uniforme, con su incorporación a una profundidad de $15 \mathrm{~cm}$ y uso de cubierta plástica durante 8 días, posterior a esto se retira, airea y riega durante 2 días (Neshev, G., 2008). Los tratamientos basados en paquetes comerciales orgánicos mediante el uso de microorganismos biocontroladores y biopreparados pertenecientes a las empresas: T1 Bioseb con sus productos Ballus - 25ml•100L(Bacilos sp.), Tricomix - 10 y 20ml•100L (tricodermas), Actimax $1.5 \mathrm{ml} \cdot 100 \mathrm{~L}$ (bacterias de distintos géneros y especies, con características termofílicas y mesofílicas) y bioles (Biol - 200ml•100L); T2 Agroinnovación con sus productos AGROSOLUTION (bacilos), Hongo Primacide (Hirsutella rhossiliensis + Arthrobotrys oligospora + Acremonium butyri), Carbon Answer (Fuentes de carbono y electrolitos), Bio N Liven (Enzimas + Co-enzimas + Exo-enzimas), Trichoderma, Gliocladium, Arthrobotrys, aplicados semanalmente mediante drench, durante el ciclo de cultivo según el paquete comercial especifico determinado para la finca por las empresas Bioseb y Agroinnovación. Los tratamientos basados en la técnica de Biosolarización con sus diferentes dosis fueron: T3 $(2 \mathrm{~kg} \bullet \mathrm{m}-2$ de material vegetal + $0.5 \mathrm{~kg} \cdot \mathrm{m}-2$ de gallinaza), T4 $(4 \mathrm{~kg} \cdot \mathrm{m}-2$ de material vegetal $+2 \mathrm{~kg} \cdot \mathrm{m}-2$ de gallinaza), T5 (5kg•m-2 de gallinaza). El material orgánico se incorpora a una profundidad entre $20-30 \mathrm{~cm}$, después cubierto con una lámina plástica por 4 semanas y posteriormente riego por aspersión por 2 días (Coello et al. 2010).

\section{RESULTADOS}

Mediante el ADEVA, se detectó diferencias altamente significativas $(p \leq 0,05)$, entre los tratamientos dentro de las variables evaluadas, por lo cual se procedió a aplicar la prueba LSD al 5\%, a fin de determinar los rangos de significancia entre los tratamientos.

Tabla 1. Comparativa entre media de las variables productivas evaluadas en el estudio según la prueba LSD 5\%.

\begin{tabular}{ccccc}
\hline Tratamiento & $\begin{array}{c}\text { No. brotes } \\
\text { por planta }\end{array}$ & $\begin{array}{c}\text { Mortalidad } \\
(\%)\end{array}$ & $\begin{array}{c}\text { No. tallos } \\
\text { por planta }\end{array}$ & $\begin{array}{c}\text { Peso del } \\
\text { tallo }(\mathbf{g})\end{array}$ \\
\hline T0 & $12.640 \mathrm{bc}$ & $4.602 \mathrm{~b}$ & $7.420 \mathrm{~b}$ & $26.523 \mathrm{a}$ \\
T1 & $14.840 \mathrm{a}$ & $5.286 \mathrm{~b}$ & $8.816 \mathrm{a}$ & $22.757 \mathrm{~b}$ \\
T2 & $13.830 \mathrm{ab}$ & $7.400 \mathrm{~b}$ & $8.794 \mathrm{a}$ & $22.961 \mathrm{~b}$ \\
T3 & $14.875 \mathrm{a}$ & $8.271 \mathrm{ab}$ & $8.810 \mathrm{a}$ & $22.274 \mathrm{~b}$ \\
T4 & $11.115 \mathrm{c}$ & $12.624 \mathrm{a}$ & $7.799 \mathrm{ab}$ & $25.072 \mathrm{a}$ \\
T5 & $11.410 \mathrm{c}$ & $8.147 \mathrm{ab}$ & $7.481 \mathrm{~b}$ & $26.909 \mathrm{a}$ \\
$\boldsymbol{p}$ - valor & 0.000367 & 0.0367 & 0.0311 & 0.000225 \\
\hline
\end{tabular}

Medias con una letra común no son significativamente diferentes. $(p \leq 0,05)$.

Se reportó, el mayor número de brotes por planta a los 40 días posterior al pinch, en los tratamientos T3 y T1 con las respectivas medias 14.875 y 14.840 brotes planta ${ }^{-1}$, ubicados dentro del rango de significancia (a); mientras que T5 y T4 con sus medias 11.410 y 11.115 , dentro del rango (c), fueron los tratamientos con menor número de brotes. 
La menor mortalidad expresada en porcentaje presentó el tratamiento T0, T1 y T2 con las respectivas medias $4.602,5.286$ y $7.400 \%$, ubicando dentro del rango de significancia (a); mientras que T4 con su media $12.624 \%$, dentro del rango (b), presentó la mayor mortalidad en porcentaje.

El mayor número de tallos por planta fue alcanzado por los tratamientos T1, T3 y T2 con las respectivas medias $8.816,8.810$ y 8.794 tallos planta ${ }^{-1}$, ubicándose dentro del rango de significancia (a); mientras que T0 y T5 con sus

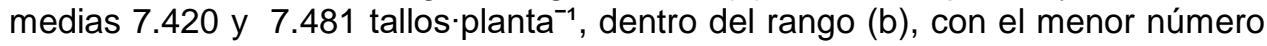
de tallos por planta.

El mayor peso por tallo presentaron los tratamientos T5, T0 y T4 con las respectivas medias $26.909,26.523$ y $25.072 \mathrm{~g}$ tallo $^{-1}$; por el contrario T1, T2 y T3 con sus medias $22.757,22.961$ y $22.274 \mathrm{~g} \cdot$ tallo $^{-1}$, dentro del rango de significancia (b), con el menor peso de tallo.

\section{DISCUSIÓN}

Dentro de la variable número de brotes por planta a los 40 días posterior al pinch, destacan T1 y T3 con el mayor número, pudiendo deberse en T1, a la acción de los microorganismos aplicados. Tricoderma sp., que se relaciona a la formación de un ambiente favorable para el desarrollo radical, lo que aumenta la tolerancia de la planta al estrés, estimulan el crecimiento y desarrollo, además de aumentar los rendimientos de los cultivos (Neshey y Labrada, 2008). Mientras que en T3 con menor cantidad de materia orgánica, dentro de la Biosolarización, no estuvo expuesto al estrés causado por altas cantidades. El exceso de humedad y la disminución de la aireación del suelo, un efecto de las altas cantidades de materia orgánica (García et al. 2009).

Para la variable mortalidad expresada en porcentaje, T0, T1 y T2 manifiestan el menor porcentaje, en T1 y T2 presumiendo la acción de los microorganismos aplicados dentro de los tratamientos. Trichoderma sp., posee efectos antagonistas sobre los patógenos edáficos (Harman, 2006). El género Bacillus es ampliamente relacionado al control de fitopatógenos, al poseer varios mecanismos de acción (Villarreal-Delgado et al. 2018). Mientras que en T0 se asume, el efectivo control sobre varios fitopatógenos edáficos por parte del fumigante Dazomet (Fao, 2008).

Para la variable número de tallos por planta destacan, los tratamientos T1, T2 y T3. En el caso de T1 y T2 se puede atribuir a los efectos benéficos añadidos de los microorganismos aplicados. Trichoderma spp. tiene la capacidad de colonizar las raíces, establecer relaciones simbióticas, promover el crecimiento y desarrollo (Harman, G. E. 2011). Mientras que T3 se presume una adecuada cantidad de materia orgánica aplicada, que evitó problemas relacionados con altas cantidades, en especial en etapas iniciales del cultivo. El exceso de humedad y la disminución de la aireación del suelo, son un efecto del altas cantidades de materia orgánica (García et al. 2009).

Dentro de la variable peso por tallo resaltan los tratamientos T0, T4 y T5. Respecto a T0, se presume el efecto de la relación fuente demanda, ya que al menor 
número tallos, estos pueden presentan mayor área foliar y peso. La acumulación de materia seca, depende del tamaño del área foliar, de la tasa a la cual funcionan las hojas y el tiempo que el follaje persiste (Tekalign y Hammes, 2005). Mientras que en el caso de T4 y T5, se presume que la alta cantidad de materia orgánica permitió una mayor disponibilidad de nutrientes durante el ciclo de cultivo. La práctica de la Biosolarización mejora el estado nutricional del suelo (Ros et al., 2002).

\section{CONCLUSIONES}

De los tratamientos basados en Biosolarización se determina, como el mejor tratamiento T3 $(2 \mathrm{~kg} \cdot \mathrm{m}-2$ de material vegetal $+0.5 \mathrm{~kg} \cdot \mathrm{m}-2$ de gallinaza $)$, al presentar un mayor número de brotes por planta y número de tallos por planta.

Dentro de los tratamientos basados en paquetes comerciales orgánicos T1 (Bioseb), se presenta como el mejor tratamiento, al presentar un mayor número de brotes por planta y número de tallos por planta, con el mismo rango de significancia que T3, a más de esto posee un menor índice de mortalidad.

\section{REFERENCIAS}

1. Agrios GN. (1998). Fitopatología. Control de las Enfermedades de las Plantas. Trad. M Guzmán Ortiz, Grupo Noriega de Editores, Limusa, México, 193-194.

2. Barres MT, Bello A, Jordá C, Tello J. (2006). La Eliminación del Bromuro de Metilo en la Protección de Cultivos como Modelo Mundial para la Conservación del Medio Ambiente. Univ. Almería, MAPA, Madrid, 515 pp.

3. Coello A, Trujillo L. Perera S. (2010). Informe de la parcela demostrativa sobre distintos métodos de biodesinfección de suelos. Servicio de Agricultura del Cabildo de Tenerife, 8 pp. Disponible en: http://www.agrocabildo. org/publicaciones_detalle.asp?id=254

4. Fao (2010), Biopreparados para el manejo sostenible de plagas y enfermedades en la agricultura urbana y periurbana. Disponible en: http://www.fao.org/3/aas435s.pdf

5. Fao. (2008). Food and Agriculture Organization of the United Nations.

Disponible en:

fao.org:http://www.fao.org/fileadmin/templates/agphome/documents/Methyl_Br omide/Methyl_bromide07.pdf, 83pp.

6. Harman, G. E. (2011), Multifunctional fungal plant symbionts: new tools to enhance plant growth and productivity. Disponible en: https://nph.onlinelibrary.wiley.com/action/showCitFormats?doi=10.1111\%2Fj.1 469-8137.2010.03614.x

7. Harman, G. E. (2006). Overview of Mechanisms and Uses of Trichoderma spp., Phytopathology 96: 190-194.

8. Neshev, G., (2008). Major soil-borne phytopathogens on tomato and cucumber in Bulgaria, and methods for their management. En: Labrada (Ed.) Alternatives to remplace methyl bromide for soil-borne pest control in east and central Europe. Manual. FAO/UNEP. Rome, Italy. pp 94 
9. Oelhaf RC. (1978). Organic Agriculture: Economic and Ecological Comparisons with Conventional Methods. J Wiley (Ed.), New York, $271 \mathrm{pp}$.

10. PROCHILE. (2017). ECUADOR, Ficha de Mercado. Disponible

en: https://www.prochile.gob.cl/wp-

content/uploads/2017/08/FMP_Ecuador_Bulbos_Lilium_2017.pdf

11. Tekalign, T. and P.S. Hammes. (2005). Growth and productivity of potato as influenced by cultivar and reproductive growth II. Growth analysis, tuber yield and quality. Scientia Horticulturae 105 (1): 29-44.

12. Tello JC, Bello A. (1994). El suelo como ente vivo. La rizosfera, los hongos y los nematodos fitopatógenos en la «memoria del suelo». I Cong. SEAE, 28-29 sept., Toledo, 506-516.

13. Tello, J., \& Bello, A. (2002). Plastics in the disinfection of agriculture land Plasticulture. 50-71.

14. Villarreal-Delgado, M., Villa-Rodríguez, E., Cira-Chávez, L., Estrada-Alvarado, M., Parra-Cota, F., \& de los Santos-Villalobos, S. (2018). El género Bacillus como agente de control biológico y sus implicaciones en la bioseguridad agrícola. Revista Mexicana De Fitopatologẽ-A, Mexican Journal Of Phytopathology, 36(1). Disponible en: :http://dx.doi.org/10.18781/R.MEX.FIT.1706-5 